\title{
Relative profiling of L-tryptophan derivatives from selected edible mushrooms as psychoactive nutraceuticals to inhibit P-glycoprotein: a paradigm to contest blood-brain barrier
}

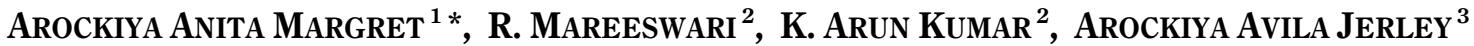 \\ ${ }^{1}$ Department of Biotechnology and Bioinformatics, Bishop Heber College, Tiruchirappalli, Tamil Nadu, India \\ ${ }^{2}$ Department of Biotechnology, National Centre of Excellence (MHRD) Thiagarajar College, Madurai, Tamil Nadu, India \\ ${ }^{3}$ Department of Zoology, Holy Cross College, Tiruchirappalli, Tamil Nadu, India
}

\begin{abstract}
Depression is a mental illness and is considered to be a global threat. It is designated as burden of disease. There is therefore an intense need to improve the therapeutic response of antidepressants. India beholds a wide fraction (Agaricus bisporus and Pleurotus ostreatus) as a vital source of non-hallucinogenic indole compounds. The amino acids L-tryptophan and 5-hydroxytryptophan (5-HTP) are precursors of serotonin. 5-HTP is a potential antidepressant that can cross the blood-brain barrier (BBB) at a high rate and is converted into serotonin more efficiently. Drug delivery across this blockade remains a challenge due to the stimulation of efflux pump receptors called permeability glycoprotein (P-gp). This work reports a comparative phytochemical assay and profiling of nonhallucinogenic tryptophan metabolites using HPLC from two organic extracts of edible mushrooms. The efficacy of the eluted compounds was authenticated as P-gp inhibitors with in vitro and in silico studies. The following four derivatives were obtained from the methanol and ethanol extracts of the mushrooms: 5-hydroxy-L-tryptophan (5HTR), 5-hydroxy tryptamine (5-HT), L-tryptophan (L-Trp), and tryptamine (TA). In vitro and molecular docking studies targeting P-gp (minimum energy: -64.38 and $-83.93 \mathrm{kcal} / \mathrm{mol}$, respectively) substantiated the ability of mushroom-derived metabolites to facilitate drug delivery in the brain. This study verified that mushrooms containing non-hallucinogenic metabolites can act as psychoactive nutraceuticals that are significant for enhancing mental health. The high therapeutic efficacy, these mushrooms can serve as ideal neurological drug leads to fortify treatment for mental illness.
\end{abstract}

Key words: depression, blood-brain barrier, mushrooms, L-tryptophan, psychoactive nutraceuticals, permeability glycoprotein

\section{Introduction}

Depression is a communal illness and is considered a threat for normal human existence, with more than 300 million people affected worldwide annually (GBD, 2016). It is a prolonged mental discomfort that affects the quality of life (Mayor, 2015). Severe health infirmities both physically and mentally with intense health disruptions can ultimately lead to suicidal tendencies. According to the global report of WHO, 800000 people die due to suicide every year (one person every $40 \mathrm{~s}$ ), and women showing depressive symptoms are more inclined to attempt suicide than men (Choo et al., 2019). Suicide is considered as the second leading cause of death among persons aged $15-29$ years globally (WHO, 2014 ), and in 2016 , it was the $18^{\text {th }}$ leading cause of death (Whiteford et al., 2016). Although a wide variety of antidepressants are commercially available, a large percentage of individuals in low- and middle-income coun-

\footnotetext{
* Corresponding author: Department of Biotechnology and Bioinformatics, Bishop Heber College, Tiruchirappalli - 620017, Tamil Nadu, India; e-mail: anitamargret@gmail.com
} 
tries are unable to receive treatments for mental disorders (Wang et al., 2018). There are more than 60 forms of major depressive disorders with varying combinations of symptoms, which makes it challenging to diagnose and treat these disorders (Stahl, 2013). Moreover, many people are unaware of the disease they have, and they continue to live with the psychological symptoms. Hence, there is an urgent need to provide solutions and remedies to eradicate this massive menace.

Although depression can be treated with antidepressant drugs and psychotherapies, adverse effects can aggravate the health condition of a patient (Vivek and Sharma, 2016; Khushboo, 2017). Thus, an approach that facilitates drug delivery to the targeted infirmity and is devoid of side effects is highly recommended. Drug efflux remains a challenge for neurological drugs that bypass the blood-brain barrier (BBB), which decreases the transcellular transport of drugs into the microvessels of brain endothelial cells (Bors and Erdo, 2019). P-glycoprotein (P-gp, multidrug resistance (MDR1) protein ) is a member of the $\mathrm{ABC}$ (ATP-binding cassette) transporter family expressed in many tissues and barriers (e.g., gut, lung, BBB) and secretory epithelia (e.g., kidney, bile ducts, blood-CSF barrier) (De Bock et al., 2016). P-gp is widely known as an efflux transporter, and it prevents the entry of blood-derived materials into the brain, thereby also limiting the central distribution of drugs that are beneficial to treat central nervous system (CNS) diseases (Sivandzade and Cucullo, 2018). Therefore, it is essential to find a natural resource with lesser side effects that can target depression by facilitating drug efflux mechanism and functioning as P-gp inhibitors. There are several vital nutrients known as psychoactive nutraceuticals that influence the neural functions; they target neurobiological pathways and may be used as efficient antidepressants (Gertsch, 2017). One such unique biocomponent is the essential amino acid tryptophan and its hydroxylated form 5-hydroxytryptophan (5-HTP) which enhance serotonergic transmission in the CNS. Moreover, tryptophan competes with other large neutral amino acids to penetrate the BBB (Birdsall, 1998; Hoglund, Overli and Winberg, 2019).

Psilocybe azurescens is a psilocyin mushroom, which is commonly termed as magic mushroom or psychedelic mushroom. It belongs to the polyphyletic group of fungi that contains important hallucinogenic compounds such as psilocybin and psilocin (Madsen et al., 2019). The mycochemical components of these mushrooms possess hallucinogenic properties and can induce succession of hallucinations among depressed patients that can cause perceptual anomalies (Jalal, 2018). Hallucinations are generally considered to have serious adverse effects. However, edible mushrooms containing active non-hallucinogenic indole compounds can act as an effective alternative antidepressant (Muszynska and SułkowskaZiaja, 2015a; Jo et al., 2014). Mushroom-derived indole compounds have the potential to become new drug leads for developing psychosomatic therapeutics for various psychiatric disorders. This study focused on investigating the presence of L-tryptophan and its derivatives among the widely available edible mushrooms (Agaricus bisporus and Pleurotus ostreatus) in vitro and in silico assays were also conducted to determine whether L-tryptophan derivatives can function as an effective neurological drug with a therapeutic efficacy of deactivating the efflux protein P-gp to overcome the BBB.

\section{Materials and methods}

\section{Chemicals}

Dulbecco's modified Eagle's medium (DMEM) and rhodamine 123 were purchased from Sigma-Aldrich (Merck). The standard amino acid along with all other chemicals and solvents were of analytical reagent grade and were obtained from HiMedia Laboratories Pvt. Ltd., Mumbai, India.

\section{Collection of plant material and sample preparation}

The mushroom samples (A. bisporus and P. ostreatus) were collected from a local organic farm in Madurai, Tamil Nadu, India. The fruiting bodies were cleaned, dried under shade for 7 days, and grinded to fine powder.

\section{Preparation of extracts}

The powdered fruiting bodies of both the mushrooms $(25 \mathrm{~g})$ were placed in $500 \mathrm{ml}$ conical flask and were extracted with $200 \mathrm{ml}$ of methanol and ethanol as separate extraction solvents. The suspensions were allowed to sediment for $24 \mathrm{~h}$ with occasional manual agitation of the flask at room temperature. After the completion of extraction, the supernatant was filtered through a Whatman ${ }^{\circledR}$ grade 1 qualitative filter paper (Sigma-Aldrich). The filtrates were then concentrated in vacuum at $40^{\circ} \mathrm{C}$ and stored at $4^{\circ} \mathrm{C}$ for further use (Song et al., 2010). 


\section{Quantitative phytochemical screening}

The quantitative screening of phytochemicals was performed according to standard biochemical procedures. The preliminary tests for methanol and ethanol extracts were performed to confirm the presence of alkaloids, flavonoids, glycosides, phenols, proteins and amino acids, saponins, steroids, volatile oils, and terpenoids following the procedures reported by Tiwari et al. (2011).

\section{HPLC analysis}

The prepared extracts were subjected to HPLC analysis to assay the presence of L-tryptophan and its derivatives. The assay was adopted from the procedure developed by Kysilka and Wurst (1985) with minor modifications. The former protocol included fluorometric and electrochemical analyses along with UV detection. In the present study, the test was conducted using a UV detector alone at the wavelength of $280 \mathrm{~nm}$ ( $\lambda$ max) using a Hitachi apparatus (Merck, Japan) with a C-18 end-capped silica column (dimensions: $150 \mathrm{~mm} \times 4.6 \mathrm{~mm} \times 5 \mu \mathrm{m}$ particle size) and thermostated between room temperature of $28^{\circ} \mathrm{C}$ and $40^{\circ} \mathrm{C}$. The solvent system selected was methanol/water/tetrahydrofuran $(15: 14: 1 \mathrm{~mol} / \mathrm{l})$ with a flow rate of $1 \mathrm{ml} / \mathrm{min}$.

\section{Cell line and culture conditions}

The human BBB cell line hCMEC/D3 (Weksler et al., 2013) was maintained in DMEM supplemented with 5\% fetal bovine serum, $2 \mathrm{mM}$ L-glutamine, $44 \mathrm{mM} \mathrm{NaHCO}_{3}$, $22.7 \mathrm{mM}$ glucose, $10 \mathrm{ml} / 1$ non-essential amino acid solution, $100 \mathrm{U}$ penicillin-G, and $100 \mu \mathrm{g} / \mathrm{ml}$ streptomycin. The cells were cultivated in $5 \% \mathrm{CO}_{2}$ under a humidified atmosphere at $37^{\circ} \mathrm{C}$. The cells were grown on 12 -well plates at the density of 37000 cells $/ \mathrm{cm}^{2}$.

\section{Functional assay for P-gp using rhodamine}

P-gp activity was assessed by determining intracellular accumulation of rhodamine 123 in hCMEC/D3. The assay is based on the inhibitory potential of the compounds that estimates the polarity and release of the fluorescent dye rhodamine 123 , which is a substrate of P-gp (Haseloff et al., 2005). Cells were incubated at $37^{\circ} \mathrm{C}$ with $5.25 \mu \mathrm{M}$ rhodamine 123 for $30 \mathrm{~min}$ in the presence or absence of organic extracts of mushrooms [ethanol and methanol extracts / A. bisporus and $P$. ostreatus $(1 \mathrm{ml})$ ]; L-tryptophan $(10 \mathrm{mg} / \mathrm{ml})$ was used as a positive control. The cultured cells were washed thrice in phosphate-buffered saline and lysed in distilled water for $1 \mathrm{~min}$. The intracellular levels of rhodamine 123 were detected using a fluorescence microplate reader (BioTek, USA; excitation wavelength at $485 \mathrm{~nm}$, emission wavelength at $538 \mathrm{~nm}$ ). The data were expressed as percentage of rhodamine 123 accumulation compared to that in non-exposed control cells randomly set at $100 \%$.

\section{Statistical analysis}

Statistical analysis was performed using Statistical Package for Social Sciences (SPSS) version 17.0. The experimental data with 3 replicates $(r=3)$ are expressed as mean \pm standard deviation (SD). Differences at $P<0.05$ were considered to be statistically significant.

\section{Molecular docking}

In silico molecular docking of six authoritative compounds (L-tryptophan, O-benzyl psilocybin, serotonin, psilocybin, sertraline, and tryptamine, N-[4-hydroxyhydrocinnamoyl]) - Table 1 - gainst the targeted protein $\mathrm{P}$-gp was carried out using the iGEMDOCK (version 2.1) graphical-automatic drug design system for docking, screening and post-analysis program.

\section{Preparation of ligands}

The 3D structures of the abovementioned six authoritative compounds, which are considered as ligands to counteract the target P-gp protein, were retrieved and downloaded in SDF format and converted to PDB format using the online tool (cactus.nci.nih.gov/translate) SMILES translator. The compound structures were energy minimized and considered for docking studies.

\section{Preparation of protein target}

The crystallographic coordinates for the P-gp target protein were retrieved from the Protein Data Bank (PDB ID 4M1M). The binding site of the target was prepared, the energy-minimized compounds were imported, and the water molecules were removed. The docking sites were enhanced by selecting one specific chain in the protein structure. Generally, during the preparation of the protein, the water $\left(\mathrm{H}_{2} \mathrm{O}\right)$ molecules were deleted. However, the water molecules that are coordinated with metals and connected between the ligand-protein were retained to facilitate a specific interaction. Several challenges such as alpha-carbon positions and structures 
Table 1. Selected compounds for molecular docking assay

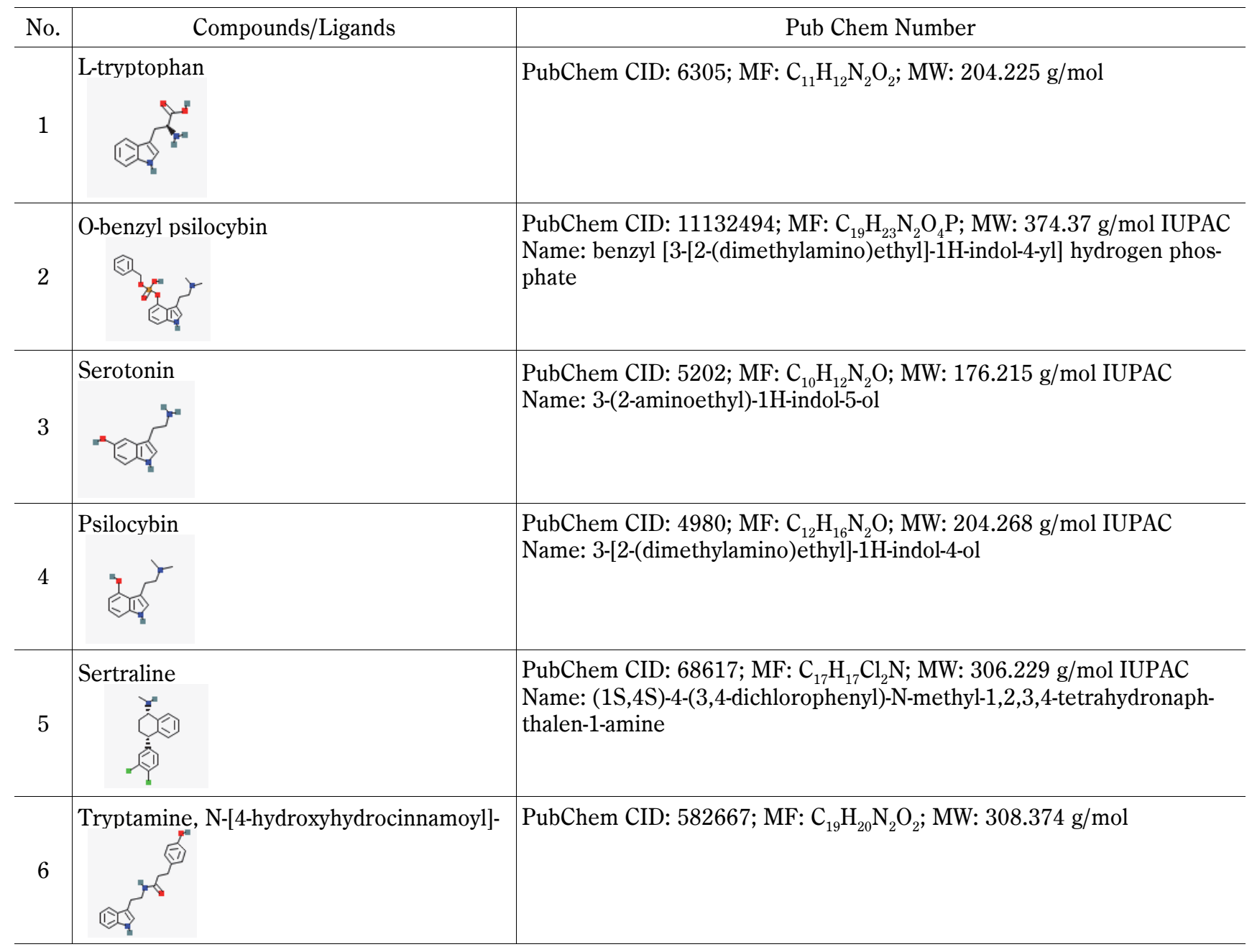

with missing loops, individual domains, or subunits from a larger molecule were perceived during downloading the protein structures in PDB format, which were repaired by adjusting the protein, metal ions, and cofactors. The structure-forming bonds from the ligand or a cofactor to a protein metal were deleted by adjusting the ligand bond orders and formal charges. Minimization was performed to restrain the input protein coordinates by a selected RMSD tolerance.

The docking protocol consisted of 25 generations per ligand and the population size of 100 random individuals. All the docking conformations were performed twice using a genetic evolutionary algorithm, and the fitness of the docked structures was calculated. The hydrophobic preference and electrostatic preference were set to 1.00. The binding site of the target was identified at the distance of $8 \AA$. The empirical scoring function of iGEMDOCK was estimated as: Fitness $=$ vdW $+\mathrm{H}$ bond + Elect.
Here, vdW is van der Waal energy and $\mathrm{H}$ bond and Elect are hydrogen bonding energy and electrostatic energy, respectively.

\section{Results and discussion}

\section{Phytochemical studies}

The phytochemical assay performed in both $A$. bisporus and $P$. ostreatus revealed the presence of bioactive components. The phytochemical active compounds were qualitatively analyzed, and the results are presented in Table 2. This screening process showed the presence of alkaloids, glycosides, saponins, phenolic compounds, tannins, amino acids, flavonoids, and terpenoids. In the methanol extract of $A$. bisporus, six groups of phytocompounds (alkaloids, saponins, terpenoids, amino acids, flavonoids, and glycosides) exhibited a high degree of precipitation. In the methanol 
Table 2. Phytochemical analysis of edible mushrooms in relavent organic solvents

\begin{tabular}{c|l|c|c|c|c}
\hline \multirow{2}{*}{ No. } & \multirow{2}{*}{ Phytochemical } & \multicolumn{2}{c|}{ Agaricus bisporus } & \multicolumn{2}{c}{ Pleurotus ostreatus } \\
\cline { 3 - 6 } & & ethanol & methanol & ethanol & methanol \\
\hline 1 & saponins & +++ & +++ & + & + \\
\hline 2 & tannins & ++ & + & +++ & ++ \\
\hline 3 & phenolic compounds & ++ & ++ & +++ & +++ \\
\hline 4 & terpenoids & ++ & +++ & + & ++ \\
\hline 5 & steroids & + & - & + & + \\
\hline 6 & volatile oil & +++ & +++ & +++ & ++ \\
\hline 7 & flavonoids & +++ & +++ & +++ & +++ \\
\hline 8 & glycosides & +++ & ++ & + & ++ \\
\hline 9 & alkaloids & +++ & +++ & + & +++ \\
\hline 10 & amino acids & +++ & +++ & +++ & +++ \\
\hline
\end{tabular}

Legend: (+++) - abundant; (++) - moderate; (-) - absent

extract of $P$. ostreatus, four phytocompound groups (alkaloids, terpenoids, amino acids, and flavonoids) were present in an increased amount with a higher rate of precipitation and turbidity. Steroids were present in both the mushrooms but were absent in the methanol extract of $A$. bisporus. This result agreed with the study of Egwim and Elem (2011) who revealed the presence of varying quantities of alkaloids, flavonoids, saponins, and tannins with the absence of anthraquinones and steroids in different wild mushrooms. Compared to the methanol extract, the ethanol extract of both mushrooms showed the presence of various compounds. Similar to the methanol extract, the ethanol extract of $A$. $b i$ sporus showed increased presence of alkaloids, glycosides, saponins, amino acids, flavonoids, and volatile oils. Saponins, terpenoids, and alkaloids showed an average presence in the ethanolic extracts of $P$. ostreatus. Phytocompounds are generally considered as bioactive substances with therapeutic properties, and the presence of vital components in the ethanol extract of $A$. bisporus enhances its medicinal properties. The preliminary phytochemical analysis optimized the finest solvents that exhibited the presence of a high number of phytocompounds. Considering the two solvents, the highest numbers of phytocompounds were identified in the methanol extract of $P$. ostreatus and in the ethanol extract of $A$. bisporus. Both mushrooms showed the presence of flavonoids and amino acids with a high level of precipitation indicated as $(+++)$. Flavonoids and flavo- noid-rich foods have broad neuroprotective potential against neurotoxic exposure, can suppress neuroinflammation, and promote cognitive functions (Bakoyiannis et al., 2019). Some groups of dietary flavonoids (anthocyanins, cyanidin-3-rutinoside, and pelargonidin-3-glucoside) impart color and have the potency to overcome the BBB (Youdim et al., 2004; Ayaz et al., 2019). Furthermore, two specific flavonoids [kaempferol-3-monoglucoside (Astragaline) and malvidin-3-O-glucoside (OENIN)] are efficacious P-gp inhibitors that can traverse the BBB (Margret et al., 2019). Hence, the presence of flavonoids in the tested edible mushrooms substantiates their ability to enhance cellular and molecular functions of the brain.

\section{Detection of the presence of tryptophan and its derivatives in the extracts by HPLC analysis}

HPLC is a popular tool used in qualitative and quantitative analysis of multifarious constituents. It is considered to be a prolific technique for the efficient separation of organic compounds that can be modified into lead drug molecules (Petrova and Sauer, 2017). The presence of tryptophan and its derivatives in the extracts of edible mushrooms was detected by HPLC. Under the optimized conditions, the standard peaks of 5-hydroxy-Ltryptophan (5HTR), 5-hydroxy tryptamine (5-HT), L-tryptophan (L-Trp), and tryptamine (TA) were eluted at 2.3, 3.4, 29.8, and $32.9 \mathrm{~min}$, respectively (Fig. 1A). HPLC profiles of the test solvents were compared with 

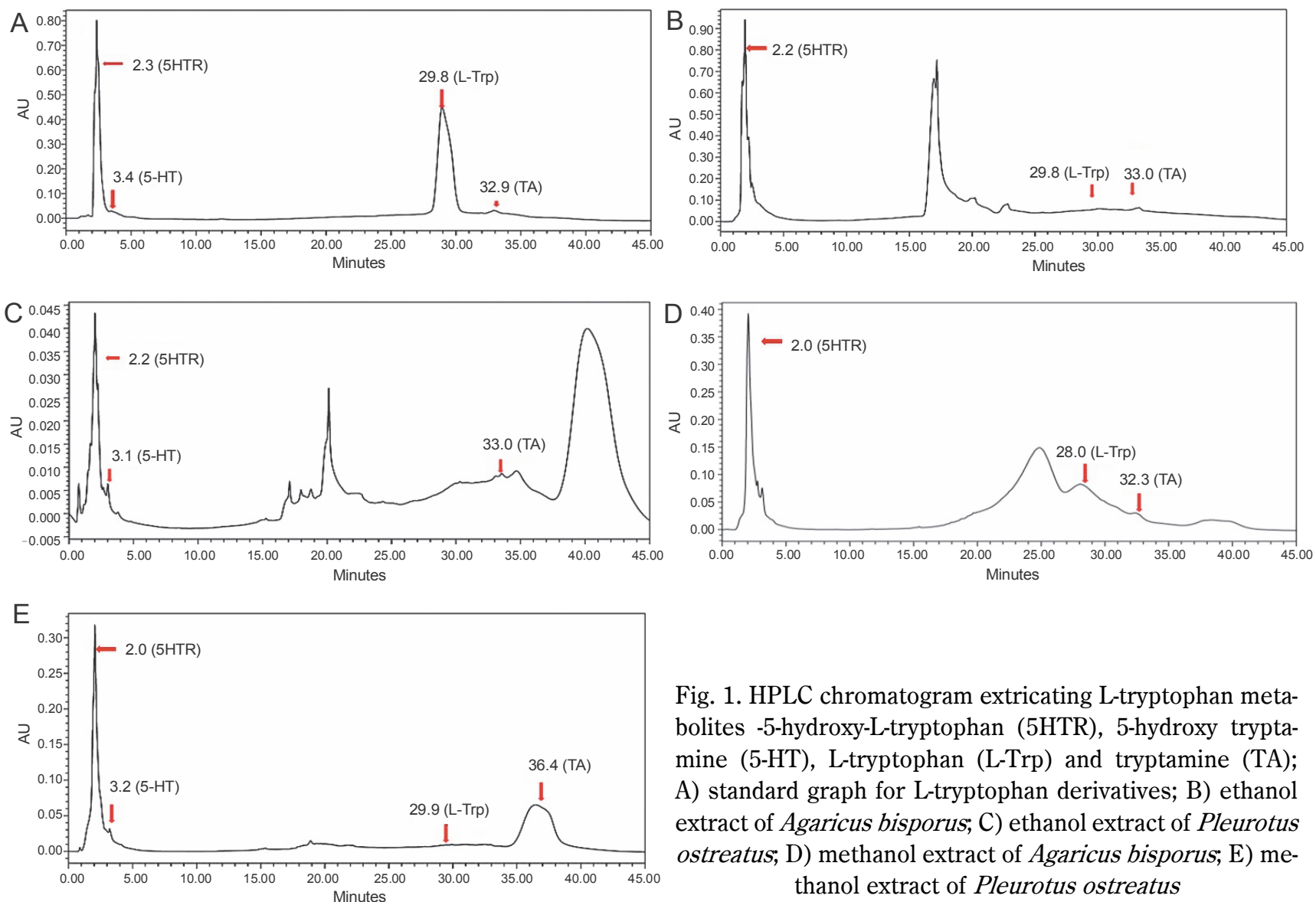

Fig. 1. HPLC chromatogram extricating L-tryptophan metabolites -5-hydroxy-L-tryptophan (5HTR), 5-hydroxy tryptamine (5-HT), L-tryptophan (L-Trp) and tryptamine (TA); A) standard graph for L-tryptophan derivatives; B) ethanol extract of Agaricus bisporus; C) ethanol extract of Pleurotus ostreatus; D) methanol extract of Agaricus bisporus; E) methanol extract of Pleurotus ostreatus

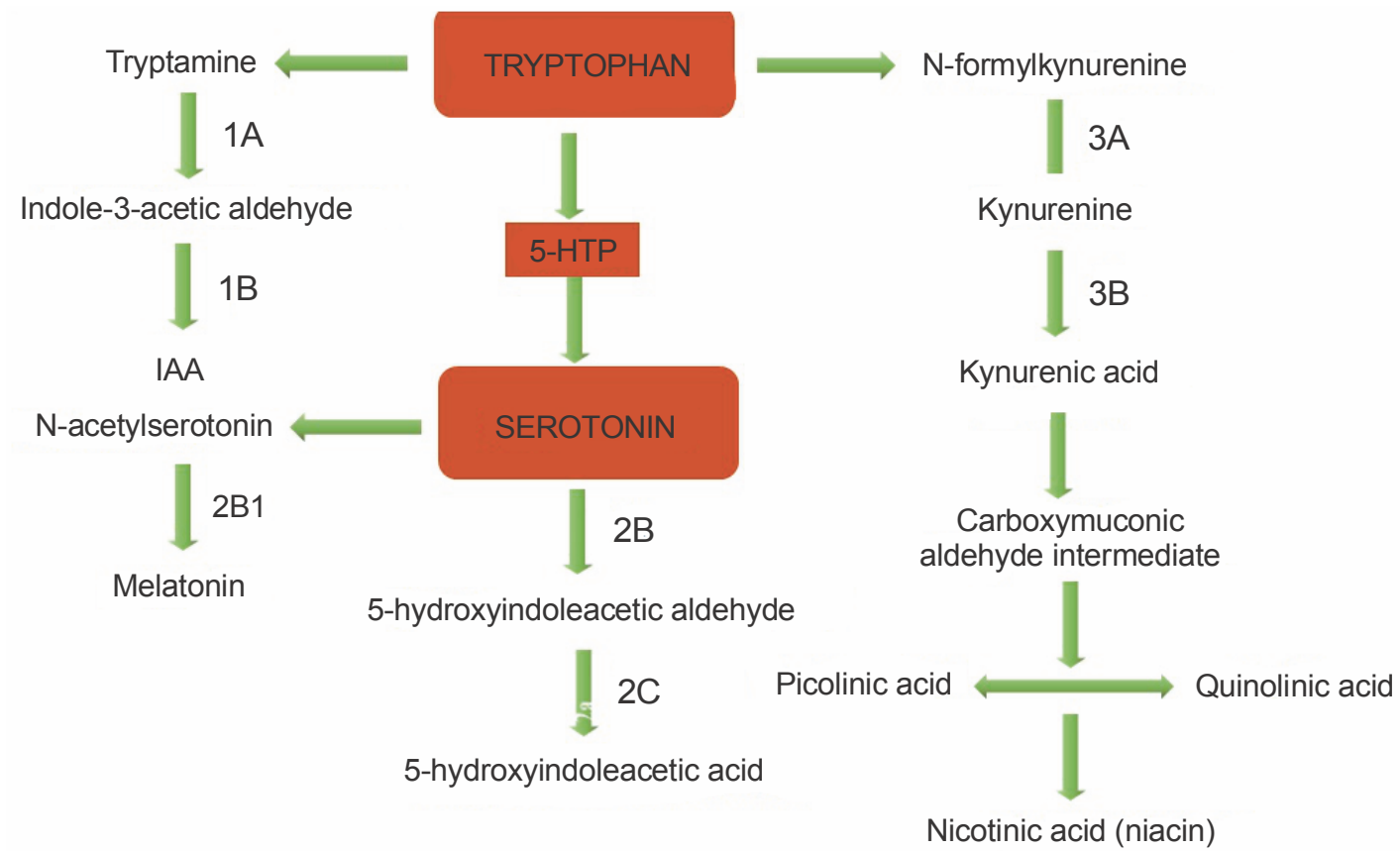

Fig. 2. Metabolic pathway of tryptophan (adapted from Muszyńska et al., 2015) 1) L-tryptophan decarboxylase, 1A) deaminase tryptamine, 1B) aldehyde dehydrogenase, 2) tryptophan hydroxylase, 2A) aromatic amino acid decarboxylase, 2B) monoaminoxidase, 2C) aldehyde dehydrogenase, 2A1) serotonin N-acetylase, 2B2) O-metyltransferase 5-hydroxyindole, 3) formylkynureninase, $3 \mathrm{~A}$ ) formamidase, $3 \mathrm{~B}$ ) kynurenine aminotransferase 
the standard chromatographs (Fig. 1B-E). The methanol extract of $A$. bisporus (Fig. 1D) extracted all four derivatives with specific retention times [5HTR $(2.0 \mathrm{~min})$, 5-HT (3.1 min), L-Trp (28.0 min), and TA (32.3 min)], and the eluted peak of L-tryptophan was broad with a distinct increased peak area. The ethanol extract of A. bisporus (Fig. 1B) lacked 5-HT but showed the presence of 5HTR, L-Trp, and TA at retention times of 2.2, 29.8 , and $33.0 \mathrm{~min}$, respectively. The ethanol extract of $P$. ostreatus did not exhibit the presence of L-tryptophan (Fig. 1C) but showed the prevalence of 5-HTR, 5-HT, and $\mathrm{TA}$ with relative retention times of $2.2,3.1$, and $33.0 \mathrm{~min}$, respectively. However, the methanol extract of $P$. ostreatus showed peaks of 5-HTR (2.0 min), 5-HT (3.2 $\mathrm{min})$, and L-Trp (29.9 $\mathrm{min})$ along with an anonymous peak at $36.4 \mathrm{~min}$ (close to the standard retention time value of tryptamine). These findings correlated well with the results of Muszynska and Sułkowska-Ziaja (2012b) where the presence of 5-HTR was found in different varieties of Basidiomycota (Macrolepiota procera). Muszynska et al. (2013) further reported 5HTR as an intermediate metabolite of L-tryptophan, which is a direct precursor in serotonin synthesis (Fig. 2). Therefore, 5-HTR deserves much attention and should be considered for further in vitro assay to confirm its pharmacological efficacy. Thus, the HPLC assay facilitated the detection of tryptophan derivatives and its metabolites that are present in the edible mushrooms.

\section{Rhodamine 123 accumulation assay to determine $\boldsymbol{P}$-gp inhibition}

Passive diffusion of the fluorescent dye rhodamine 123 across biological membranes is the principle mechanism underlying the assaying of the efficiency of P-gp inhibition (Le Vee et al., 2015). This assay was performed in the $\mathrm{hCMEC} / \mathrm{D} 3$ cell line in the presence and absence of inhibitors (ethanol and methanol extracts of $A$. bisporus and $P$. ostreatus, L-tryptophan) so that the rate of inhibition could be determined. The percentage of dye accumulation is inversely proportional to inhibition efficiency; thus, as dye accumulation increases, there is a relative inactivation of the P-gp-mediated efflux mechanism (Sivandzade et al., 2019). The cell lines with standard 5HTR showed a high intensity of dye accumulation than the ethanol and methanol extracts of both mushrooms. P. ostreatus showed a greater effect in this dye accumulation assay than $A$. bisporus (Fig. 3). Although

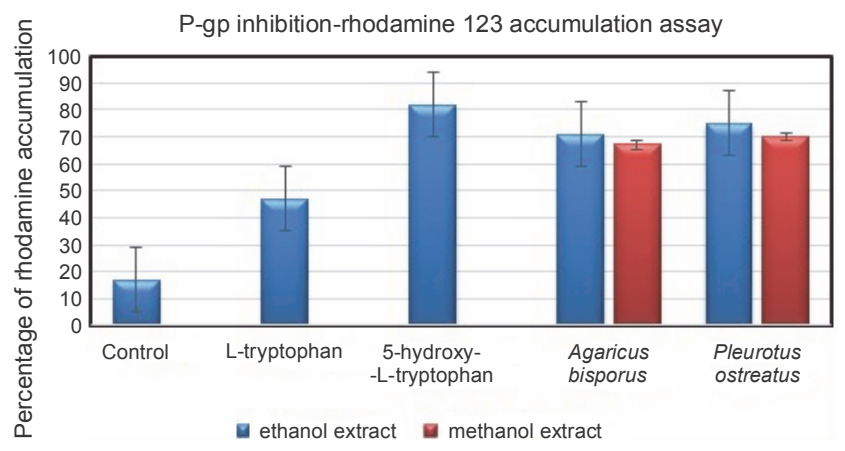

Fig. 3. Accumulation of rhodamine 123 in hCMEC/D3 cells; control; cells exposed to $5.25 \mu \mathrm{M}$ rhodamine 123 for $30 \mathrm{~min}$ at $37^{\circ} \mathrm{C}$ in the absence of inhibitors; data are expressed as fluorescence arbitrary unit (FAU)/mg protein and are the means \pm SEM of three independent experiments; $P<0.05$ when compared to control

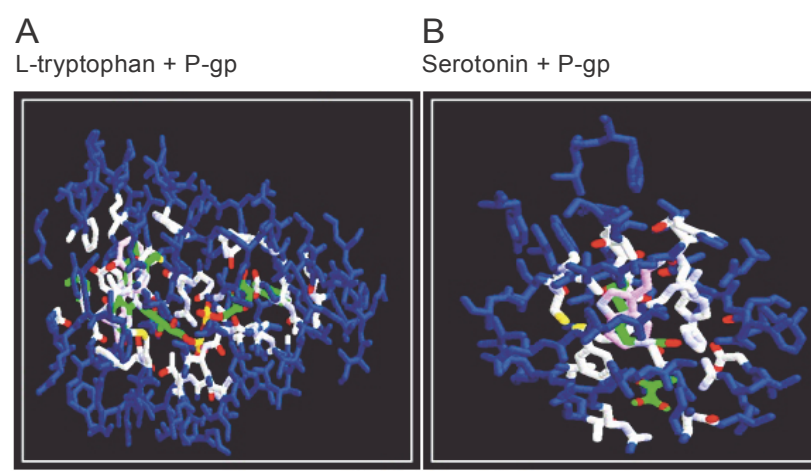

C Psilocin + P-gp
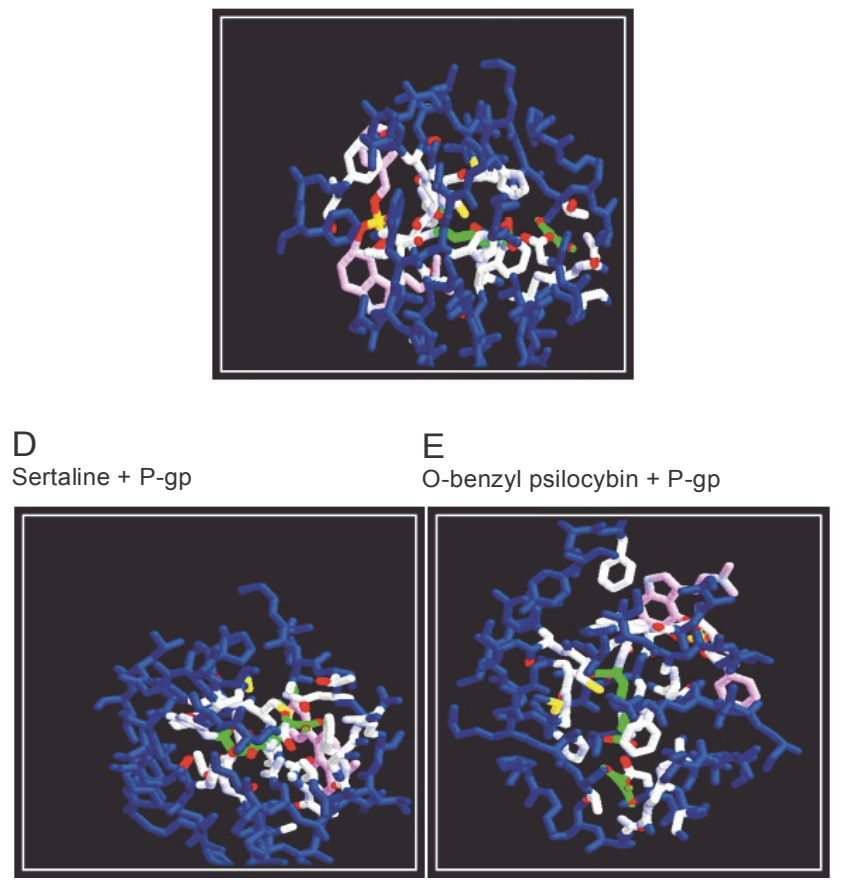

Fig. 4. Interaction of P-glycoprotein with the test ligands 
Table 3. Hit list of compounds targeting P-gp

\begin{tabular}{l|c|c|c|c}
\hline \multicolumn{1}{c|}{ Phytocompounds } & Energy & VDW & H Bond & Elect \\
\hline L-tryptophan & -64.38 & -34.58 & -29.80 & 0 \\
\hline Serotonin & -83.93 & -60.93 & -23.00 & 0 \\
\hline O-benzyl psilocybin & -69.61 & -53.64 & -15.97 & 0 \\
\hline Psilocin & -53.14 & -46.14 & -7.00 & 0 \\
\hline Sertraline & -55.68 & -52.18 & -3.50 & 0 \\
\hline
\end{tabular}

L-Trp resulted in P-gp inhibition, the accumulation of dye was lesser $(47 \%)$ than that achieved with 5 HTR (82\%). To summarize the in vitro assay, the inactivation of drug efflux was efficient with a greater permeability. All the test samples that allowed dye accumulation were considered to be competent when compared with the control (17\%). Although L-Trp is an essential amino acid, it cannot be endogenously synthesized and requires to be supplied externally with food-derived transport molecules (Palego et al., 2016) Furthermore, in addition to L-Trp, there are various other supplementary amino acids (tyrosine, phenylalanine, valine, leucine, and isoleucine) with an increased contribution in food (Fernstrom et al., 2013). The impact of these amino acids competes with the absorption and transportation where the distribution of L-Trp is decreased relatively into the CNS. Compared to 5HTR, L-Trp acts as a precursor and facilitates various other metabolic activities such as serotonin and kynurenine synthesis, protein production, and formation of organic compounds such as niacin (Puglisi-Allegra and Andolina, 2015; Sandi and Haller, 2015). 5HTR shows a promising drug efflux mechanism against P-gp and can overcome the BBB (ZamanianDaryoush et al., 2020). On the basis of these findings, L-Trp is a better drug efflux inhibitor as it facilitates the synthesis of serotonin.

\section{Molecular docking studies against the efflux protein}

An in silico assay was performed using the molecular docking tool iGEMDOCK $v$ 2.1. to determine the best compound that can serve as a lead drug molecule by inhibiting the efflux protein P-gp. iGEMDOCK is a computational tool used for identifying pharmacological interactions and virtual screening (Hsu et al., 2011). The targeted protein was docked with six ligands consisting of a standard antidepressant (Sertraline), mushroom-derived commercial antidepressants (O-benzyl psilocybin and psilocin) along with L-Trp and its metabolites (serotonin and 5HTR). Sertraline is considered as a positive control and can comparatively substantiate the therapeutic efficacy of test ligands against the target protein. Five compounds (O-benzyl psilocybin, psilocin, L-tryptophan, serotonin, and 5HTR) were found to interact efficiently with the target protein exhibiting energy minimization scores (Table 3). The minimized energy value of the docked compounds determined the inhibitory efficiency, where the standard drug sertraline interacted with an energy value of $-55.68 \mathrm{kcal} / \mathrm{mol}$. Subsequently, the test compounds serotonin, L-Trp, O-benzyl psilocybin, and psilocin and its derivatives inhibited P-gp with minimum binding energies of $-64.38,-83.93,69.61$ and $53.14 \mathrm{kcal} / \mathrm{mol}$. The outcomes strongly supported P-gp inhibition with a very good binding efficiency between receptor and ligands with the calculated energy values. Considering the docking scores, the highest minimum energy value was shown by serotonin. The synthesis of serotonin in the brain is mainly dependent on the availability of L-Trp and the transformation of 5HTR. The BBB remains a challenge for the penetration of serotonin, but $5 \mathrm{HTR}$ can easily cross the BBB and overcome its mechanism (Waku et al., 2010). Serotonin uses a vital receptormediated mode of action to cross the BBB and exerts its effects in target cells through at least 14 receptors, where 13 of them belong to the G-protein-coupled receptors (GPCRs) superfamily (Suominen, Piepponen and Kostiainen, 2015). On the basis of the association with the receptors and the utilization of various secondary messengers, serotonin was shown to be adequately competent to inhibit P-gp (Kang et al., 2009). The receptormediated mode of serotonin to overcome the BBB requires extensive in vitro and in vivo studies to confirm its specific mechanism. The docking study did not exhibit any inhibitory action of the non-hallucinogenic metabolite TA, N-[4-hydroxyhydrocinnamoyl], with the target protein. TA is a monoamine alkaloid structured with an indole ring and is considered to have neuromodulation capacity (Ray, 2010). Monoamines have limited access to enter the brain through the BBB, but some compounds (such as methamphetamine, 4-methylenedioxymethamphetamine (MDMA), cocaine, and nicotine) with hallucinogenic properties are prevented to enter through the BBB (Kousik, Napier and Carvey, 2012). Psychedelic molecules such as TAs consist of neutral charge and can act against the receptor sites present in 
the physiological barrier (Bradford et al., 2011; Callaghan et al., 2012). Therefore, TA, N-[4-hydroxyhydrocinnamoyl], being a non-hallucinogenic molecule did not inhibit P-gp and also did not show its competency against the BBB. This in silico study proves the ability of the non-hallucinogenic tryptophan derivatives as an effective inhibitor of the efflux protein P-gp to enable the drug to cross the BBB.

\section{Conclusions}

This study reports the therapeutic efficacy of edible mushroom extracts ( $A$. bisporus and $P$. ostreatus) as a vital source of non-hallucinogenic tryptophan derivatives with the potential to inhibit P-gp in the brain. The preliminary phytochemical studies performed for both the mushroom extracts validated the presence of vital sources of amine and lipid components. The HPLC profiles of both ethanol and methanol extracts of mushrooms showed the presence of the following four significant tryptophan derivatives: 5HTR, 5-HT, L-Trp, and TA. 5HTR was one of the critical eluents identified in both mushroom extracts and showed the maximum accumulation of the rhodamine 123 dye in the P-gp inhibition assay. The molecular docking studies conferred the inhibitory efficiency of L-Trp and 5HTR with a minimum energy value against the targeted efflux protein P-gp. This was more efficient than the standard antidepressants sertraline, O-benzyl psilocybin, and psilocin. Because it is an essential amino acid, tryptophan can be readily taken into the body by consuming edible mushrooms. Thus, this study provides a sound foundation to prove the resource of L-tryptophan derivatives in edible mushrooms and can serve to conduct clinical trials that can help to treat neurological illnesses.

\section{Acknowledgments}

The authors are grateful to the support offered by the management of Bishop Heber and Holy Cross College, Tiruchirappalli, Tamil Nadu, India. The instrumentation facility provided by Thiagarajar College, Madurai, Tamil Nadu, India, is also notably acknowledged.

\section{References}

Ayaz M., Sadiq A., Junaid M., Ullah F., Ovais M., Ullah I., Ahmed J., Shahid M. (2019) Flavonoids as prospective neuroprotectants and their therapeutic propensity in aging associated neurological disorders. Front. Aging Neurosci. 11: 155-159.
Bakoyiannis I., Daskalopoulou A., Pergialiotis V., Perrea D. (2019) Phytochemicals and cognitive health: are flavonoids doing the trick? Biomed. Pharmacother. 109: 1488-1497.

Birdsall T.C. (1998) 5-hydroxytryptophan: a clinically-effective serotonin precursor. Altern. Med. Rev. 3(4): 271-280.

Bors L.A., Erdo F. (2019) Overcoming the blood-brain barrier. Challenges and tricks for CNS drug delivery. Sci. Pharm. 87(1): 6 .

Bradford S.T., Stamatovic S.M., Dondeti R.S., Keep R.F., Andjelkovic A.V. (2011) Nicotine aggravates the brain postischemic inflammatory response. Am. J. Physiol. Heart Circ. Physiol. 300: H1518-H1529.

Callaghan R.C., Cunningham J.K., Sykes J., Kish S.J. (2012) Increased risk of Parkinson's disease in individuals hospitalized with conditions related to the use of methamphetamine or other amphetamine-type drugs. Drug. Alcohol. Depend. 120: 35-40.

Choo C.C., Harris K.M., Ho R.C. (2019) Prediction of lethality in suicide attempts: gender matters. Omega. 80(1): 87-103.

De Bock M., Van Haver V., Vandenbroucke R.E., Decrock E., Wang N., Leybaert L. (2016) Into rather unexplored terrain-transcellular transport across the blood-brain barrier. Glia. 64: 1097-1123.

Egwim E., Elem R. (2011) Proximate composition, phytochemical screening and antioxidant activity of ten selected wild edible Nigerian mushrooms. AJFN. 1(2): 89-94.

Fernstrom J.D., Langham K.A., Marcelino L.M., Irvine Z.L.E., Fernstrom M.H., Kaye W.H. (2013) The ingestion of different dietary proteins by humans induces large changes in the plasma tryptophan ratio, a predictor of brain tryptophan uptake and serotonin synthesis. Clin. Nutr. 32(6): 1073-1076.

GBD 2016 Disease and injury incidence and prevalence collaborators. (2017) Lancet 390(10100): 1211-1259.

Gertsch J. (2017) Cannabimimetic phytochemicals in the diet - an evolutionary link to food selection and metabolic stress adaptation? Br. J. Pharmacol. 174: 1464-1483.

Haseloff R.F., Blasig I.E., Bauer H.C., Bauer H. (2005) In search of the astrocytic factor(s) modulating blood-brain barrier functions in brain capillary endothelial cells in vitro. Cell. Mol. Neurobiol. 25 (1): 25-39.

Hoglund E., Overli O., Winberg S. (2019) Tryptophan metabolic pathways and brain serotonergic activity: a comparative review. Front. Endocrinol. 10: 158.

Hsu K.C., Chen Y.-F., Lin S.-R., Yang J.M. (2011) iGEMDOCK: a graphical environment of enhancing GEMDOCK using pharmacological interactions and post-screening analysis. BMC. Bioinform. 12(1): S1-S33.

Jalal B. (2018) The neuropharmacology of sleep paralysis hallucinations: serotonin $2 A$ activation and a novel therapeutic drug. Psychopharmacology 235(11): 3083-3091.

Jo W.S., Hossain M.A., Park S.C. (2014) Toxicological profiles of poisonous, edible, and medicinal mushrooms. Mycobiology 42(3): 215-220.

Kang K., Park S., Kim Y.S., Lee S., Back K. (2009) Biosynthesis and biotechnological production of serotonin derivatives. Appl. Microbiol. Biotechnol. 83(1): 27-34. 
Khushboo Sharma B. (2017) Antidepressants: mechanism of action, toxicity and possible amelioration. J. Appl. Biotechnol. Bioeng. 3(5): 437-448.

Kousik S.M., Napier T.C., Carvey P.M. (2012) The effects of psychostimulant drugs on blood brain barrier function and neuroinflammation. Front. Pharmacol. 3: 121.

Kysilka R., Wurst M. (1985) High performance liquid chromatographic determination of hallucinogenic indoleamins with simultaneous UV photometric and voltametric detection. J. Chromatogr. 320(2): 414-420.

Le Vee M., Jouan E., Stieger B., Lecureur V., Fardel O. (2015) Regulation of human hepatic drug transporter activity and expression by diesel exhaust particle extract. PLoS One 10(3): e0121232.

Madsen M.K., Fisher P.M., Burmester D., Dyssegaard A., Stenbæk D.S., Kristiansen S., Johansen S.S., Lehel S., Linnet K., Svarer C. et al. (2019) Psychedelic effects of psilocybin correlate with serotonin $2 A$ receptor occupancy and plasma psilocin levels. Neuropsychopharmacol. 44(7): 1328-1334.

Margret A.A., Dhayabaran V., Suvaithenamudhan S., Parthasarathy S. (2019) Analysing the antidepressant and drug efflux competence of Clitoria ternatea L. as P-glycoprotein inhibitor to facilitate blood brain barrier. Act. Sci. Biol. 41(1): e46629.

Mayor S. (2015) Persistent depression doubles stroke risk despite treatment, study finds. BMJ (Clinical Research ed.) 350: h2611.

Muszynska B., Sułkowska-Ziaja K. (2015a) Impact of food processing on non-hallucinogenic indole derivatives in edible mushrooms. [in:] Processing and impact on active components in food. Ed. Preedy V., San Diego, CA. Academic Press: 55-62.

Muszynska B., Sułkowska-Ziaja K. (2012b) Analysis of indole compounds in edible Basidiomycota species after thermal processing. Food Chem. 132(1): 455-459.

Muszynska B., Sułkowska-Ziaja K., Ekiert H. (2013) Analysis of indole compounds in methanolic extracts from the fruiting bodies of Cantharellus cibarius (the Chanterelle) and from the mycelium of this species cultured in vitro. JFST 50(6): 1233-1237.

Palego L., Betti L., Rossi A., Giannaccini G. (2016) Tryptophan biochemistry: structural, nutritional, metabolic, and medical aspects in humans. J. Amino Acids 2016: 8952520.

Petrova E., Sauer K. (2017) High-performance liquid chromatography (HPLC)-based detection and quantitation of cellular c-di-GMP. Meth. Mol. Biol. 1657: 33-43.

Puglisi-Allegra S., Andolina D. (2015) Serotonin and stress coping. Behav. Brain Res. 277: 58-67.

Ray T.S. (2010) Psychedelics and the human receptorome. PLoS One 5(2): e9019.

Sandi C., Haller J. (2015) Stress and the social brain: behavioural effects and neurobiological mechanisms. Nat. Rev. Neurosci. 6: 290.
Sivandzade F., Bhalerao A., Cucullo L. (2019) Analysis of the mitochondrial membrane potential using the cationic JC-1 dye as a sensitive fluorescent probe. Bio-protocol 9(1): e3128.

Sivandzade F., Cucullo L. (2018) In-vitro blood-brain barrier modeling: a review of modern and fast-advancing technologies. J. Cereb. Blood Flow Metab. 38: 1667-1681.

Song W.Y., Ku K.H., Choi J.H. (2010) Effect of ethanol extracts from red pepper seeds on antioxidative defense system and oxidative stress in rats fed high-fat, highcholesterol diet. Nutr. Res. Pract. 4(1): 11-15.

Stahl S.M. (2013) Stahl's essential psychopharmacology: neuroscientific basis and practical applications. 4th ed. New York: Cambridge University Press.

Suominen T., Piepponen T.P., Kostiainen R. (2015) Permeation of dopamine sulfate through the blood-brain barrier. PLoS ONE 10(7): e0133904.

Tiwari P., Kumar B., Kaur M., Kaur G, Kaur H. (2011) Phytochemical screening and extraction: a review. Intern. Pharmaceut. Sci. 1(1): 98-106.

Vivek Kumar G., Sharma B. (2016) Modulations of mammalian brain functions by antidepressant drugs: role of some phytochemicals as prospective antidepressants. Evid. Based Med. Practice 1(3): 1-12.

Waku T., Shiraki T., Oyama T., Maebara K., Nakamori R., Morikawa K (2010) The nuclear receptor PPARY individually responds to serotonin- and fatty acid-metabolites. EMBO J. 29(19): 3395-3407.

Wang S.M., Han C., Bahk W.M., Lee S.J., Patkar A.A., Masand P.S., Pae C.U. (2018) Addressing the side effects of contemporary antidepressant drugs: a comprehensive review. Chonnam Med J. 54(2): 101-112.

Weksler B., Romero I.A., Couraud P.O. (2013) The human brain endothelial cell line hCMEC/D3 as a human blood brain barrier model for drug transport studies. Fluids Barriers CNS 10(1): 10-16.

Whiteford H.A., Ferrari A.J., Vos T. (2016) Challenges to estimating the true global burden of mental disorders. Lancet Psychiatry 3: 402-403.

World Health Organization (accessed July 01, 2020); The top 10 causes of death. 2014. http://www.who.int/media centre/factsheets/fs310/en/index2.html.

Youdim K.A., Qaiser M.Z., Begley D.J., Rice-Evans C.A., Abbott N.J. (2004) Flavonoid permeability across an in situ model of the blood-brain barrier. Free. Radic. Biol. Med. 36(5): 592-604.

Zamanian-Daryoush M., Gogonea V., DiDonato A.J., Buffa J.A., Choucair I., Levison B.S., Hughes R.A., Ellington A.D., Huang Y., Li X.S. et al. (2020) Site-specific 5-hydroxytryptophan incorporation into apolipoprotein AI impairs cholesterol efflux activity and high-density lipoprotein biogenesis. J. Biol. 295(15): 4836-4848. 